\title{
Improved Variance Cut Algorithm for Light Source Estimation in Augmented Reality
}

\author{
Divya Kamboj \\ Computer Science Department \\ Amity University \\ Noida, India
}

\author{
Wankui Liu \\ School of optoelectronics \\ Beijing institute of Technology \\ Beijing, China
}

\begin{abstract}
Consistent illumination plays very important role in efficient integration of virtual objects into real world scene. Real time photometric registration requires efficient detection and segmentation of the brighter regions in an image to provide better light source detection and thus efficient illumination of virtual objects is possible. The paper presents a technique for light source sampling of images taken through a fish-eye Camera. These images taken through this Camera are sampled using improved variance-cut algorithm. The technique helps to detect the brighter region efficiently. With deferred rendering, some experimental results demonstrate that the proposed method produces plausible AR visualization
\end{abstract}

\section{Keywords}

AR(augmented reality), illumination, median-cut algorithm

\section{INTRODUCTION}

There are many approaches used for illumination in augmented reality [1]

- Omni-directional environment maps

- Use of equipment's like mirror ball/sphere in the scene.

- Manually or semi-manually model the entire scene, including the light sources, and perform inverse rendering.

Some of the Challenges faced by illumination in augmented reality are Complex computations, inconsistent geometry of the real scenario, use of complex objects for camera calibration and dynamic changing environment. All these problems lead to inconsistent illumination and thus a challenging task to provide seamless illumination in AR.In order to estimate illumination information in real time fish eyecamerawas used. Fish eye camera provides a 180 degree view of the environment. This reduces the time for image processing as in other techniques that requires HDR images or light probes for illumination estimation. The paper proposes technique which uses fish eye camera for acquiring images and a variance-cut algorithm for sampling image to detect the brighter region for light source estimation.Section 2 gives an overview of the previous work. Section 3 describes the proposed method.Results and discussion are explained in Section 4. Section 5 gives conclusion of the paper.

\section{PREVIOUS WORK}

For real time rendering of virtual objects in real world consistent illumination is required.In the previous work many different approaches are followed to detect the of light sourceposition for efficient lightning of virtual objects

Some of the approaches followed can be classified as:

1. By using single image with known geometry[2][3][4]

2. By using HDR images[5][6][7]
3. Shadow Analysis[8][9]

4. By using direct lightning approach / Illumination detection using fish eye cameras and other video cameras using sampling algorithm[10]

5. By Using a calibration object without distance illumination assumption[11]

The techniques above require a high computational complexity and sometimes become difficult for real time illumination estimation. The previous sampling algorithm for light source estimation described in[12] [13] reduces the computational complexity but at the same time the efficiency of algorithm reduces with different intensity regions.

\section{PROPOSED METHOD}

The system comprises of a fish eye camera capturing an image which is then divided in a way that the light source information is available to render virtual objects. The pixel intensity for the bright regions has more information about the light as compared to other region. In the first step we used median cut algorithm [13] for sampling the image region.

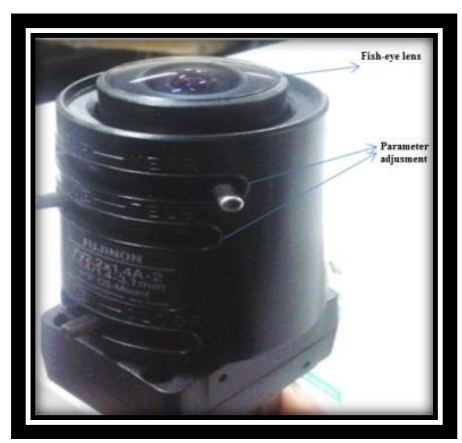

Figure1:Fish-Eye Camera

The partitioning can be done as[13]

Animage taken through a fish-eye camera is divided into the rectangular regions in latitude -longitude format into $2^{\wedge}$ nregions and each region has equal amount of light energy.

1. The image is taken as an input through s fish-eye camera.

2. The image is then divided along the longest side through the center of gravity so that the energy is equally divided.

3. Return to step 2 until number of iterations are less than $n$

4. The light sources position is then detected by finding the center of each region or centroid and then the light sources are placed at center of each 
region and the sum of pixel of a particular region gives the color of the light source

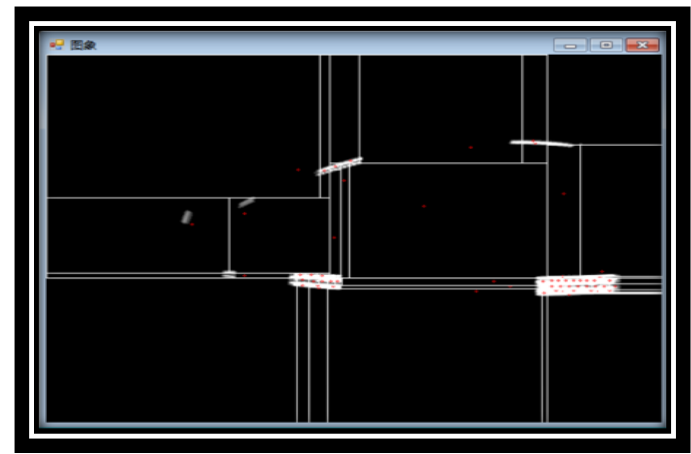

Figure 2: Showing the division of light by median -cut algorithm

The proposed algorithm when implemented using visual studio 2010 with XNA 4.0 Tool, Showed that the algorithm was able to divide the image regions for light source estimation but at the same time had some flaws

- The algorithm was unable to divide the regions having different intensities

- The algorithm divided through the brighter light source and hence was not able to divide the image regions properly.

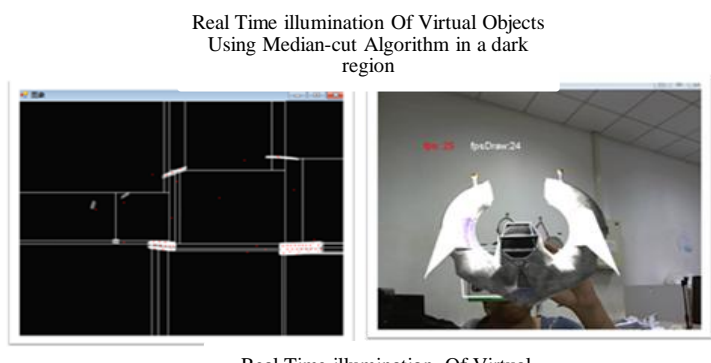
a semi-dark region
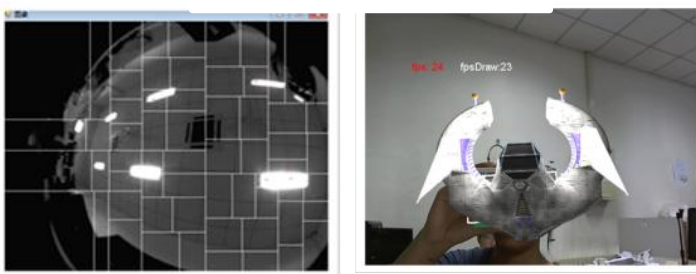

Real Time illumination Of Virtual Objects Using Median-cut Algorithm in a brighter region
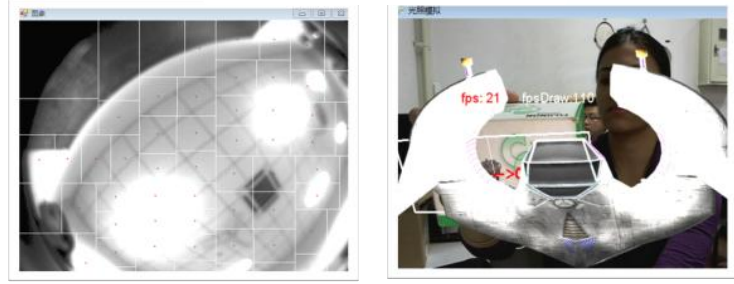

Figure3:Showing the realtime illumination of virtual objects using median cut algorithm in different light conditions

The mean calculation here gives an idea where the pixels are located and no information about how they are spread which leads to the above problem in division of regions with unequa intensity.In order to overcome this problem the variance of the pixel will be considered instead of mean of pixels. The variance of pixels will tell about how far each pixel is spread .Minimizing the area with maximum variance will lead to better estimation of light source .The algorithm calculates the variance of an image region and then find out the direction of maximum variance.The algorithm tries to minimize the variance of the sub-region of images to efficiently calculate the light source position.The Fish-eye camera is used to input a real time image of the surrounding real time environment.
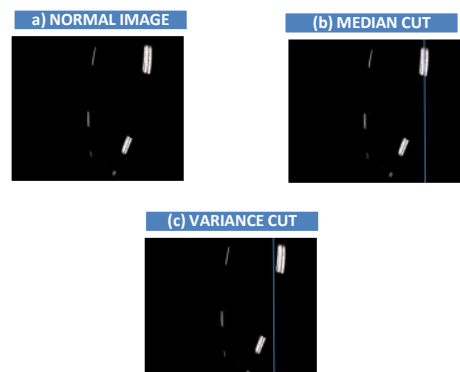

Figure4: Median and Variance cut algorithm dividing the light sources with different intensity. a) normal image (b) median- cut (c) variance-cut

\section{A.VARIANCE-CUT ALGORITHM}

1. Add the input image taken through a Fish -Eye camera

2. Subdivide the image along the longest side through the center of gravity such that the such that variance of image is minimized.

3. Repeat step 2 until number of iterations is less than $n$.

4. The light source position is detected at the centroid/center of each region.

5. The region variance can be calculated as

$$
\begin{aligned}
& \quad \text { Variance }=\sqrt{\left(\boldsymbol{\Sigma}(g-m)^{2} * p\right)} / N \\
& \mathrm{~g}=\text { Graylevel } \\
& \mathrm{m}=\text { Mean } \\
& \mathrm{p}=\text { Number of pixels in its particular graylevel } \\
& \mathrm{N}=\text { total pixels in an image } \\
& \mathrm{N}=\text { number of iteration generating } 2^{n} \text { light sources regions }
\end{aligned}
$$

Where mean can be calculated as:

Mean=sum of pixel intensity/total number of pixels

\section{B.ESTIMATION PROCEDURE}

The process of estimating the light sources using the variance cut involves the following steps:

\section{STEP 1: Image capture through a fish-eye lens}

STEP 2: Image sampling to detect the light sources using variance-cut algorithm

STEP 3: Generation of script which shows the direction of light sources

STEP 4: Creation of virtual objects in 3DS MAX 2014

STEP 5: Rendering of the scene with detected light sources 


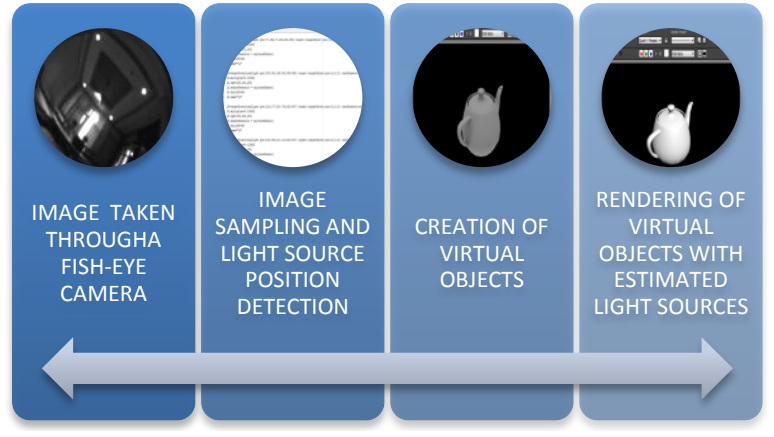

Figure 5: Showing the steps involve in illumination estimation

STEP 1:IMAGE CAPTURE THROUGH A FISH-EYE LENS Images are captured using a fish-eye camera which captures a 180 degree wide angle image and also controls the amount of incident lightwith aperture.

STEP 2:IMAGE SAMPLING TO DETECT THE LIGHT SOURCES USING VARIANCE-CUT ALGORITHM

The captured images are segmented using the variance-cut algorithm which divides the region in a way that the maximum variance is minimized which finds the bright regions in an image

\section{STEP 3:GENERATION OF SCRIPT WHICH SHOWS THE DIRECTION OF LIGHT SOURCES}

The program is implemented in MATLAB . The input image is segmented and the light source positions are estimated and a script is generated giving the direction of light sources

\section{STEP 4:CREATION OF VIRTUAL OBJECTS IN 3DS MAX 2014}

The algorithm is implemented in MATLAB, the output of program generateda scriptwhich when run in 3DS MAX software will produce the light source direction to provide a virtual environment lightning condition. Virtual objects are then created to render them with estimated light source position

\section{STEP 5:RENDERING OF THE SCENE WITH DETECTED LIGHT SOURCES}

The generated script is then run inside the 3DS MAX 2014 which show the direction oflight sources. The virtual objects are then rendered with the estimated light sources.

The light source estimation is a complex task. Real time rendering of virtual objects is also possible using a Fish-eye lens and a sampling algorithm. The 3DS software provides a view of rendering virtual objects and how they will actually look when placed in an augmented reality scene. In this paper we present the light source estimation technique that uses a fish eye camera and an algorithm for detecting light source position along with 3DS MAX to represent the results of rendering virtual objects with the estimated lights positions in the image.

\section{RESULTS AND DISCUSSION}

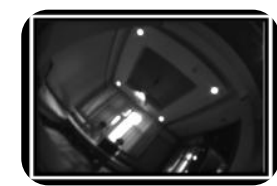

ORIGINAL IMAGE FOR LOGHT SOURCE ESTIMATION

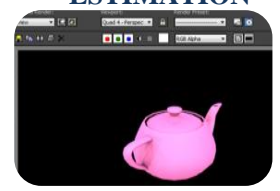

RENDERED VIRTUAL OBJECT WITH VARIANCF.CITT

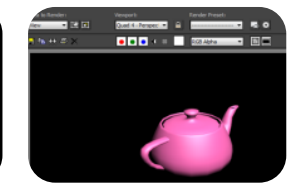

VIRTUAL OBJECT

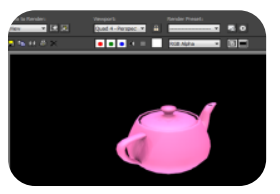

RENDERED

VIRTUAL OBJECT WITH
Figure6: Results depicting the light source rendering of virtual objects after illumination estimation

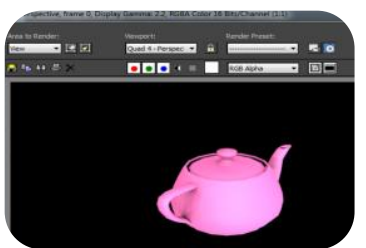

MEDIAN-CUT ALGORITHM WITH 64 LIGHTS

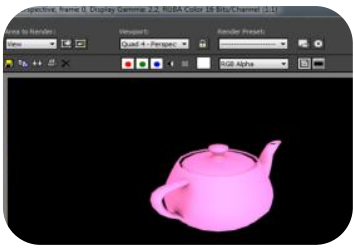

MEDIAN-CUT ALGORITHM WITH 256 LIGHTS

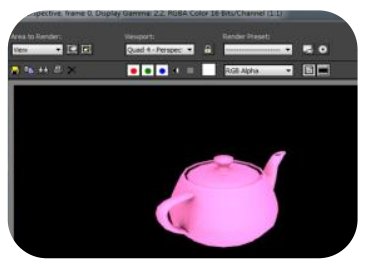

VARIANCE-CUT ALGORITHM WITH 64 LIGHTS

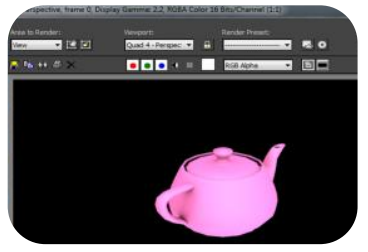

VARIANCE-CUT ALGORITHM WITH 256 LIGHTS
Figure 7: Results depicting the rendering of virtual objects in different number of light source

The input image in figure 6 is taken by a fish-eye camera and is passed as an input to the program which is implemented in MATLAB, which then generates a script file showing the direction of position of light sources in $\mathrm{n}$ iterations generating $2^{n}$ number of light sources. The generated script is then run in 3DS MAX software which produces the light sources in desired directions. The virtual objects are then rendered with these estimated directional light sources .The estimated results are compared with the median-cut algorithm which shows that with variance-cut algorithm there is better rendering of virtual objects.

Figure7 demonstrate the illumination of virtual objects under different number of light sources .The lightning effect changes with different number of light sources. From the above figure it is clear that variance-cut algorithm produces better lightning effects than median -cut algorithm 


\section{CONCLUSION\&FUTURE WORK}

Illumination in augmented reality is a complex task and faces a lot of challenges due to complex computations required for real time illumination estimation and difficulty in light source representation of dynamically changing real environment. In this paper an illumination technique was presented for light source estimation in order to illuminate the virtual objects in augmented reality. An algorithm was presented which enabled the sampling of images to detect the brighter regions for estimating the light source position. The images were taken through a Fish-eye camera which provides a wide angle of view of around 180 degree. The algorithm was able to sample the image regions for finding out the light source position to render the virtual objects. The estimated light was able to provide a realistic view of virtual object .The quality of view could be better if some shadow based algorithm would have been considered. The shadow and shading techniques will make the augmented reality scene more realistic. The properties of different types of illuminants such as multiple light sources and single light sources will be considered for better illumination detection.

\section{REFERENCES}

[1] K. Jacobs and C. LoscosClassification of Illumination Methods for Mixed Reality. In Proceedings Computer Graphics Forum, 2006

[2] K. Hara, K. Nishino, and K. Ikeuchi, "Determining reflectance and light position from a single image without distant illumination assumption," inProc. IEEE Int. Conf. Comput. Vis., 2003, vol. 1, pp. 560-567

[3]Yang Wang and DimitrisSamaras, "Estimation of Multiple Illuminants from a Single Image of Arbitrary Known Geometry". In ECCV '02 Proceedings of the 7th European Conference on Computer Vision-Part III, Springer-Verlag 2002

[4]Alexandros Panagopoulos, Chaohui Wang, Dimitris Samaras and Nikos Paragios," Illumination Estimation and Cast Shadow Detection through a Higher-order Graphical Model". In Computer Vision and Pattern Recognition (CVPR), 2011 IEEE

[5] KusumaAgusanto, Li Li, Zhu Chuangui, Ng Wan Sing, "Photorealistic rendering for augmented reality using environment illumination ".In Proceedings of the Second IEEE and ACM International Symposium on Mixed and Augmented Reality (ISMAR '03)

[6]Jonas Unger, Andreas Wenger, Tim Hawkins, Andrew Gardner, and Paul Debevec,"Capturing and rendering with incident light" . In Eurographics Symposium on Rendering: 14th Eurographics Workshop on Rendering., pages 141.149, June 2003

[7] Gibson S., Cook, J., Howard, T., and Hubbold, R., 2003,"Rapid shadow generation in real-world lighting Environments". In Proceedings: EuroGraphics Symposium on Rendering

[8]Taeone Kim, Ki-Sang Hong ."A Practical Approach for Estimating Illumination Distribution from Shadows Using a Single Image".In Wiley Periodicals, 2005

[9]Yang Wang and DimitrisSamaras ."Estimation of multiple directional light sources for synthesis of augmented reality images". In Elsevier Science ，2003.

[10] Matthias Korn, MaikStange, Andreas von Arb,Lisa
Blum, Michael Kreil, Kathrin-Jennifer

Kunze.'Interactive Augmentation of Live Images using a HDR Stereo Camera" In Journal of Virtual Reality and Broadcasting, Volume 4, 2007

[11]SeokjunLee ; Soon Ki Jung ,'Estimation of illuminants for plausible lighting in augmented reality". InUbiquitous Virtual Reality (ISUVR), 2011

[12] Jae Doug Yoo and Kwan H. Lee, "Real Time Light Source Estimation Using a Fish-Eye Lens with ND Filters".In International Symposium on Ubiquitous Virtual Reality, (C) 2008 IEEE,ISUVR.2008.

[13] P. Debevec, " A median cut algorithm for lightprobes sampling”, In poster of SIGGRAPH 2005,ACM, Los Angeles. 